\title{
ENHANCING VITALITY IN ACADEMIC MEDICINE
}

\section{FACULTY DEVELOPMENT AND PRODUCTIVITY}

\author{
Megan M. Palmer, Krista Hoffmann-Longtin, Tony Ribera, \\ Mary E. Dankoski \\ Indiana University School of Medicine \\ Amy K. Ribera, Tom F. Nelson Laird \\ Indiana University School of Education
}

The prevalence of low satisfaction and increased stress among faculty in academic medicine makes understanding faculty vitality in this field more important than ever before. To explore the contributors to and outcomes of faculty vitality, we conducted a multi-institutional study of faculty in academic medicine ( $N=1,980,42$ percent response rate). Faculty were surveyed about climate and leadership, career and life management, satisfaction, engagement, productivity, and involvement in faculty development. Analysis reveals that controlling for other factors, academic medicine faculty who participate regularly in faculty development activities are significantly more satisfied, engaged, and productive.

Compared with other faculty members across higher education, those in academic medicine face both common and unique challenges. Common 
challenges include increased calls for institutional accountability from the public and an increasingly diverse and technologically savvy student body. Academic physicians are expected to meet the same bar for promotion and tenure as other faculty across academe yet must also simultaneously meet expectations to generate clinical revenue.

Because faculty are the greatest resource of any academic institution, medical schools must recruit, retain, and advance the most talented faculty possible in order to meet the urgent and complex demands of health care education today. This includes developing new educational models to train the next generation of scientists and physicians in a rapidly shifting, political health care environment, generating research that improves health amid intense competition for funding, and developing increasingly efficient models of care delivery. The environment can be described as high stakes, competitive, fast paced, complex, and constantly changing. As such, recent research shows that faculty in academic medicine report increased stress, depression, and decreased satisfaction (Kelly, Cronin, \& Dunnick, 2007; Schindler et al., 2006). Furthermore, challenges in faculty life are often magnified for underrepresented minorities (URM) and women. URM faculty comprise less than 10 percent and women less than 36 percent of all current medical school faculty (Castillo-Page, 2012; Joliff, Leadley, Coakley, \& Sloane, 2012), and these groups are promoted and tenured at lower rates than majority or male faculty. Given the importance of faculty in creating a better future for health professions education and the strain faculty are currently under, the need to improve our understanding of faculty vitality has never before been greater.

\section{Defining Faculty Vitality}

The concept of faculty vitality has been discussed in the higher education literature since the mid-1980s. Despite multiple studies in the past twenty-five years using a variety of proxy indicators such as satisfaction or productivity (Baldwin, 1990; Bland, Seaquist, Pacala, Center, \& Finstad, 2002; Chan \& Burton, 1995; Pololi, Conrad, Knight, \& Carr, 2009; Woods, Reid, Arndt, Curtis, \& Stritter, 1997), the construct is still imprecise and lacks a predictive model (Clark, Boyer, \& Corcoran, 1985). Clark and Lewis (1985) offered this early description: "those essential, yet intangible positive qualities of individuals and institutions that enable purposeful production" (p. 176). Elsewhere faculty vitality has been defined as "faculty members' commitment to and ability to achieve both their own goals and their institution's goals" (Bland et al., 2002, p. 369). 
Over the past five years, we have developed the Faculty Vitality Survey and a resulting model of faculty vitality (Dankoski, Palmer, Nelson-Laird, Garver, \& Bogdewic, 2012). (The survey is in the chapter appendix.) In this model, faculty vitality is defined as the synergy between high levels of satisfaction, productivity, and engagement that enables faculty members to maximize their professional success and achieve goals in concert with institutional goals. Not only is it critical for faculty developers to understand what makes faculty members highly engaged, productive, and satisfied, it is also important to expand our knowledge about how faculty development programs may aid in sustaining vitality.

\section{Assessing Outcomes of Faculty Development}

Despite the importance of faculty development, assessing its outcomes can be difficult. Unfortunately, many faculty development programs track relatively simplistic metrics such as attendee satisfaction and faculty contacts, without conducting more rigorous analyses such as linking programs to student learning outcomes, assessing for learning or behavioral change, or mapping efforts to institutional measures (Birch \& Gray, 2009; Chism \& Szabo, 1997-1998). Without more sophisticated studies, faculty developers are hard-pressed to know how to best invest precious programming dollars or address questions about the value of faculty development to individual faculty members and the institution.

There is, however, a small but growing body of literature in academic medicine about faculty development assessments and outcomes. For instance, studies comparing participants and nonparticipants in parttime, off-site, cohort-based programs have shown that participants have higher academic promotion rates (Smith, Barry, Dunn, Keefe, \& Weismantel, 2006), increased collegial relationships (Morzinski \& Fisher, 2002), and increased attainment of leadership roles (Simpson, Bragg, Biernat, \& Treat, 2004). A recent literature review on faculty development initiatives designed to promote leadership had a similar conclusion: leadership development program completers often obtain new leadership positions and also self-report attitude changes and knowledge gains (Steinert, Naismith, \& Mann, 2012).

Studies of local programs (those conducted at participants' home institutions) have also shown important outcomes. One such program that combined career planning, scholarly writing, and peer mentoring yielded increased publication rates among participants (Pololi, Knight, Dennis, \& Frankel, 2002). Similarly, a program targeted toward faculty retention and academic productivity showed positive results in both of 
these areas (Morzinski \& Simpson, 2003); another program focused on scientific writing among medical school faculty was found to be effective in improving confidence, writing ability, and productivity (Dankoski et al., 2012). Many medical schools offer structured programs for junior faculty members and formal mentoring programs, both of which aid in the retention of faculty (Ries et al., 2012; Wingard, Garman, \& Reznik, 2004) and greater research productivity (Bland, Weber-Main, Lund, \& Finstad, 2005). A qualitative analysis investigated the role of a longitudinal faculty development program in the growth of an "academic identity" among health professions faculty (Lief et al., 2012). Furthermore, several programs intended to improve teaching abilities have yielded significant gains in faculty teaching skill, commitment, and confidence (Barratt \& Moyer, 2004; Berbano, Browning, Pangaro, \& Jackson, 2006; Knight et al., 2005; Pololi \& Frankel, 2005). As one way to explore faculty development outcomes, Palmer, Dankoski, Smith, Brutkiewicz, and Bogdewic (2011) compared indicators related to faculty satisfaction, mentoring, feeling valued, and career planning between two points in time during which multiple new faculty development initiatives had been launched.

Although not meeting the full definition of return on investment analyses, using more rigorous assessment methods such as those described moves faculty developers away from simply reporting reactions or satisfaction to measuring important institutional results (Bothell \& Henderson, 2004). The purpose of our study was to investigate the relationship between engagement in faculty development and the construct of faculty vitality as another approach to exploring the result of institutional investment in faculty development.

\section{Purpose}

This study examines responses from 1,980 faculty members in academic medicine to better understand faculty engagement in faculty development and the relationship between that engagement and faculty productivity. Three research questions guided this work:

1. How often do faculty in academic medicine participate in faculty development activities?

2. What topical areas are faculty members most or least likely to attend?

3. Does participating in professional development activities have an effect on faculty productivity? 


\section{Methods}

In spring 2011, faculty members at four academic medical centers participated in an administration of the Faculty Vitality Survey. The survey instrument asks faculty about their perceptions and experiences with various aspects of their work. The self-report survey contained demographic variables (gender, race/ethnicity, academic rank, track) and subscales measuring perceptions of institutional climate and leadership, career and life management, satisfaction, engagement, and productivity. The survey also included questions about the frequency of attendance at various types of faculty development programs for an analysis regarding how participation in such programs may be related to faculty vitality. Specifically, participants were asked to indicate approximately how many times over the previous academic year they had participated in professional development activities (e.g., workshops, conferences, online tutorials) related to each of the following areas: promotion and tenure, teaching and learning, research, advancement of women, and other diversity issues. The focus of this chapter is on the extent to which faculty in academic medicine engage in faculty and professional development activities.

\section{Sample}

This study was limited to academic medicine at four public medical schools in the United States. After removing cases with a large number of missing responses, the final sample consisted 1,980 faculty members in academic medicine (institutional response rates ranged from 31 percent to 78 percent). Table 6.1 shows sample characteristics.

The majority of our participants were employed full time by their institutions (93 percent, $n=1,743$ ). The majority were male (62 percent, $n=1,147)$ and white $(71$ percent, $n=1,313)$. Approximately one-sixth of the sample ( 15 percent, $n=276$ ) were Asian, and a much smaller percentage identified as black or African American ( 2 percent, $n=39$ ) or Latino or Hispanic ( 3 percent, $n=60$ ). The largest number of faculty in the sample were assistant professors (42 percent, $n=769$ ). Associate rank faculty (26 percent, $n=489$ ) and full professors (28 percent, $n=519$ ) were less represented in our sample. The majority of faculty in the sample spent 30 percent or more of their time on clinical duties (53 percent, $n=1,055)$ while 38 percent $(n=757)$ spent 30 percent or more of their time on research. Faculty in the sample spent less time on teaching (17 percent, $n=330$ ) and administrative duties (17 percent, $n=339$ ). 
Table 6.1 Descriptive Statistics of Faculty Respondents in Academic Medicine

Count

Gender

Male

Female

Employment status

Full time

Part time

Race or ethnicity

Asian

Black or African American

Latino or Hispanic

White

Other

Academic rank

Assistant

Associate

Full

Other

Percentage of faculty time spent ${ }^{a}$

Research focused

Teaching focused

Clinical focused

Administrative focused
1,147

691

1,743

122

276

39

60

1,313

161

769

489

519

72

757

330

1,055

339
Percentage

62

38

93

7

15

2

3

71

9

42

26

28

4

38

17

53

17

${ }^{2}$ Percentage who spent 30 percent or more of their time on research, teaching, clinical, or administrative duties. Since there is potential that faculty could spend more than 30 percent of their time in different areas, the total percentage exceeds 100 percent.

\section{Selected Measures and Analyses}

To answer our first research question, we examined faculty responses to the item, "To what extent are you currently engaged in faculty development activities?" We report the percentage of faculty who indicated engaging in these activities very little, some, quite a bit, or very much. For our second research question, "How often do faculty in academic medicine participate in faculty development activities?" we looked at the number of activities in which faculty participated. Faculty members were asked to indicate how many times they participated in seven specific types of professional development activities (see table 6.4 for a complete list). 
Table 6.2 Item-level Descriptive Statistics of Productivity Scales

Research productivity (alpha $=.81$ )

Participation in professional organizations in my field Translating research into practice

Securing external funding

Number of peer-reviewed publications

Number of peer-reviewed conference presentations

N Mean

SD

Teaching and service productivity (alpha $=.67$ )

Teaching evaluations

$\begin{array}{lll}1,784 & 3.28 & 1.13 \\ 1,709 & 3.04 & 1.17 \\ 1,739 & 2.72 & 1.31 \\ 1,772 & 2.93 & 1.35 \\ 1,754 & 3.02 & 1.28\end{array}$

Number of learners with whom I interact

1,723

3.74

1.00

Participation in university/department/school service

1,762

3.65

1,771

3.45

1.02

1.15

Faculty could select 0,1 to 3,4 to 6,7 to 9 , or more than 10 . To answer our third research question, "Does participating in professional development activities have an effect on faculty productivity?" we conducted a linear regression to better understand the effect engagement in faculty and professional development activities had on faculty productivity.

Two productivity scales were used in this analysis: research productivity (alpha $=.81$ ) and teaching and service productivity (alpha $=.67$ ). The research productivity scale combines five items indicating the extent to which faculty engage in research-related activities (e.g., securing external funding) and the amount of scholarship (e.g., number of peerreviewed publications). The teaching and service productivity scale combines three items capturing faculty members' self-rated performance on teaching evaluations and the extent to which they interact with learners and participate in university, department, and/or school service related to the education mission. Productivity items are described in table 6.2.

\section{Results}

Frequencies in table 6.3 suggest that most faculty members in academic medicine engage in faculty development activities at least "some." When asked about the extent to which they engage in faculty development activities, close to seven out of ten (66 percent, $n=1,143$ ) indicated they do this "some," "quite a bit," or "very much." Among this group, 28 percent $(n=480)$ reported that they engage in these activities frequently ("quite a bit" or "very much"). Approximately one-third of the sample ( 34 percent, $n=579$ ) indicated that they participated in faculty development activities very little. 
Table 6.3 Level of Engagement in Faculty Development Activities

$\begin{array}{lcc} & \text { Number } & \text { Percentage } \\ \text { Very Little } & 579 & 34 \\ \text { Some } & 663 & 38 \\ \text { Frequently } & 480 & 28 \\ \text { aCombined response options "quite a bit" and "very much." } & \end{array}$

Table 6.4 Average Yearly Participation in Professional Development by Topical Area

Topical Area

Research

Teaching and learning

Leadership

Promotion and tenure

Other diversity issues

Advancement of women

Advancement of underrepresented minority
Average Yearly Participation

3.4

3.4

1.9

1.3

0.8

0.7

0.7

Although these findings suggest that most faculty members in academic medicine engage in at least some faculty development activities, we are also interested in the focus of these activities. Table 6.4 illustrates the average participation in faculty development activities by topical area. Among a list of seven topical areas, research $(M=3.4)$ and teaching and learning ( $M=3.4$ ) had the highest average yearly participation. Activities focused on the advancement of women $(M=0.7)$, the advancement of underrepresented minorities $(M=0.7)$, and other diversity issues $(\mathrm{M}=0.8)$ had the lowest average yearly participation.

These findings provide valuable information on the type of professional development activities in which medical school faculty engage. It is not surprising, given the demands of academic medicine, that events focused on supporting faculty in their research and teaching are among the most widely attended. However, due to the quantitative nature of this study, it is unclear what might contribute to average yearly participation.

Table 6.5 shows the results of the regression analyses. Model 1 explained a significant portion of the variance in research productivity 


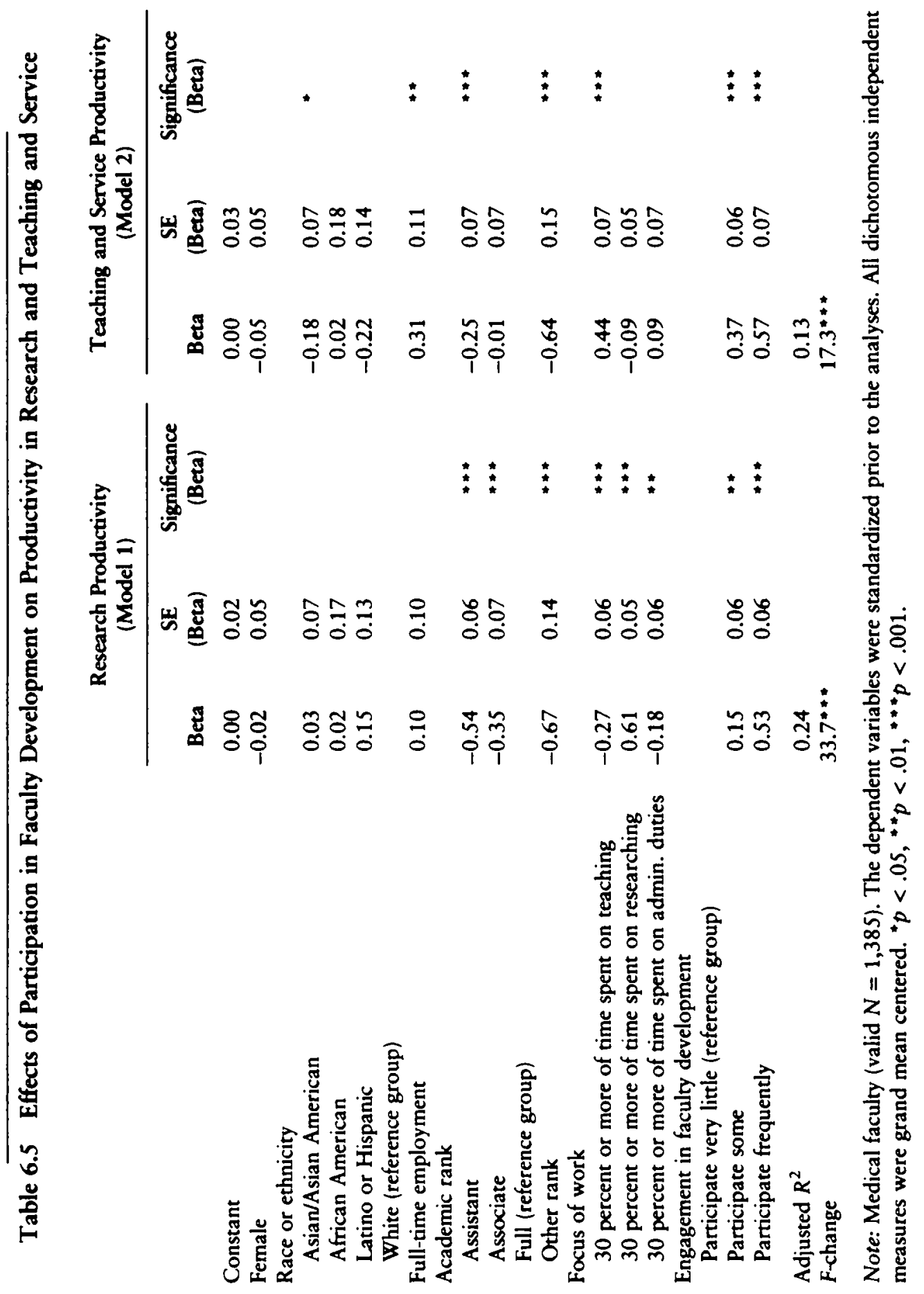


among faculty $\left(F=33.7, p<.001\right.$; adjusted $\left.R^{2}=0.24\right)$. Results suggested gender, race or ethnicity, and employment status were not significant predictors of research productivity. However, academic rank, focus of work, and, most important, engagement in faculty development were all found to be significantly related to research productivity.

Similar to previous studies on faculty vitality, full professors were more productive in research than assistant and associate faculty. Model 1 indicates that on average, assistant professor faculty scored half of a standard deviation lower and associate rank faculty scored over one-third of a standard deviation lower than full professors. Model 1 also shows a small negative effect on research productivity if faculty spent more than 30 percent of their time on teaching (beta $=-27 ; p<.001$ ) or administrative duties (beta $=-.18 ; p<.01$ ). Yet a medium positive effect on research productivity was found among faculty who spent 30 percent or more of their time on research (beta $=.61 ; p<.001$ ).

We also found a positive relationship with engagement in faculty development and research productivity. That is, faculty who "frequently" engaged in professional development produced half a standard deviation more research than those who engaged in professional development "very little." A significantly positive but smaller effect on research productivity was found among those who only participated in "some" professional development (beta $=15 ; p<.01$ ).

For faculty productivity in teaching and service, model 2 explained a significant but smaller portion of the variance $(F=17.3, p<.001$; adjusted $R^{2}=0.13$ ). After controlling for gender, race or ethnicity, employment status, rank, and focus of work, we found that participation in faculty development had a significant and positive effect on faculty productivity in teaching and service. Specifically, faculty who are frequent participants in faculty development engaged in teaching and service three-fifths of a standard deviation more than faculty who participated in faculty development "very little." The model also shows a positive significant effect on teaching and service productivity when faculty participate in only "some" faculty development activities (beta $=37 ; p<.001$ ). Other notable findings were significant differences by race/ethnicity, rank, and work focus. Compared to white faculty, Asians (beta $=-.18 ; p<.05$ ) engaged in teaching and service slightly less when controls were introduced. The model also indicated that assistant professors engaged in teaching and service slightly less than full professors. Furthermore, spending 30 percent or more time on research was not significantly related to productivity in teaching service. However, the model showed a significant positive effect (beta $=.44$; 
$p<.001)$ on productivity in teaching and service if faculty spent more than 30 percent of their time on teaching.

\section{Conclusion}

The findings from this study reveal that faculty developers, at least in academic medicine, have more work to do to reach the majority of the faculty. The fact that slightly over one-third of the respondents indicated that they participate in faculty development "very little" raises three key questions:

What should faculty developers expect in terms of the amount and level of participation in faculty development programming?

$\checkmark$ Are the current faculty development offerings not meeting the needs of nearly one-third of the faculty, which may explain their low levels of participation?

o In what ways does or could the reward structure encourage faculty to participate in development activities?

With regard to anticipated levels of participation in faculty development, there likely is not a single answer because this is dependent on institutional resources and culture. Yet it is worth having local and national conversations about what the benchmarks should be in terms of the number of faculty reached and the ideal extent of faculty involvement in faculty development activities. This is a particularly important discussion given the positive relationship between engagement in faculty development and productivity.

Faculty developers in academic medicine, and likely developers in other areas, should determine why participation levels vary. That is, are the offerings not relevant to a subgroup of faculty? Or perhaps the timing of the workshops or events doesn't work well for faculty who are responsible for taking care of a large number of patients. It could be useful for faculty developers to conduct surveys or focus groups with nonparticipants to better understand this issue.

Finally, faculty developers should examine how the reward structures encourage or discourage participation. For example, to what degree are department chairs actively inviting or discouraging faculty to participate in development activities? Similarly, are faculty recognized when they take, or are expected to take, their professional development seriously? Is language present in promotion and tenure 
documents that require that faculty document their engagement in professional development activities?

Faculty members were most likely to attend workshops and events focused on teaching and research. Activities related to diversity and the advancement of women were not as widely attended. Given the nature of this research, we cannot be certain what contributes to the level of participation in each of the areas measured. It is possible that this may have less to do with the interests of individual faculty members and more to do with the number of programs offered in these areas at each institution. With this perspective, it could be that faculty participation in programs focused on the advancement of URM faculty is low because of a lack of offerings at the institutions in this sample when compared to research or teaching and learning programs. Conversely, faculty may be less interested in or less comfortable attending workshops or events related to diversity and women's issues. More research is needed to better understand faculty interests and the amount and types of professional development activities at academic medical centers. Deeper understanding regarding this matter could assist faculty developers in better meeting the needs of the faculty and potentially provide insight into the values of the medical school faculty with regard to diversity and the advancement of women.

Faculty developers are, of course, interested in the impact of faculty development activities on outcomes that matter (in this case, productivity), and the results of this study provide good news. Based on the findings, we can conclude that not only faculty in academic medicine benefit from engagement in professional development; the institution also benefits. That is, faculty who engage in faculty development activities report greater levels of productivity. It is not difficult to argue that productivity is the coin of the realm in academic medicine. Therefore, the results of this study can assist faculty developers in demonstrating the benefit of engagement in faculty development. Furthermore, these results can be used when speaking with junior faculty, department chairs, and institutional leaders regarding why engagement in faculty development activities is important. The results of the study also provide evidence that investment in faculty development within medical schools is paying off.

More work can and should be done regarding the return on investment for faculty development. This study, focused on academic medicine, provides faculty developers with some additional insight regarding the outcomes of the work and why faculty development remains a critical piece of the equation in ensuring that each faculty member can be a success story. 


\section{Appendix: Faculty Vitality Survey, 2011, Indiana University School of Medicine}

Many of the following items inquire about your experiences, perceptions, and satisfaction with the academic environment in what you consider to be your primary unit; that is, the unit you feel most closely affiliated.

Please consider the following options and identify one as your primary unit. Response options: School, Department, Division, Regional Center, Other.

Consider your work over the last academic year. Approximately what percentage of your time was devoted to activities related to the following areas (must add to $100 \%$ )?

Teaching

Research

Patient care/clinical work

Administrative duties (including committee service)

Consider your experiences over the last academic year, and rate to what extent you agree with the following statements. Response options: Strongly agree, Somewhat agree, Neither agree nor disagree, Somewhat disagree, Strongly disagree, Not applicable/l don't know.

Colleagues are fully engaged in their work in my primary unit.

Opportunities for faculty development are offered by my primary unit.

Fair mechanisms for acknowledging achievements are in place in my primary unit.

Women have an equal opportunity for advancement as men in my primary unit.

Minority faculty are provided equal opportunities for advancement as white faculty in my primary unit.

Effective recruitment strategies are in place for attracting the best talent to my primary unit.

There is a shared vision in my primary unit.

My primary unit is comprised of a well-developed network of colleagues.

My contributions are valued by the leaders in my primary unit. Consider your experiences over the last academic year, and rate to what extent you agree with the following statements. Response 
options: Strongly agree, Somewhat agree, Neither agree nor disagree, Somewhat disagree, Strongly disagree, Not applicable/l don't know.

Effective strategies to retain productive faculty are employed by leaders of my primary unit.

Faculty achievements are often recognized by the leaders of my primary unit.

An inclusive environment is created by the leaders of my primary unit.

Conflict is effectively handled by the leaders of my primary unit. Faculty feel empowered to act by the leaders of my primary unit. My opinions are routinely solicited by the leaders of my primary unit. The leaders of my primary unit are highly regarded by others within the unit.

The leaders of my primary unit are highly regarded by others outside the unit.

The leaders of my primary unit are willing to challenge the status quo.

The leaders of my primary unit provide me guidance to improve. Consider your experiences over the last academic year, and rate to what extent you agree with the following statements. Response options: Strongly agree, Somewhat agree, Neither agree nor disagree, Somewhat disagree, Strongly disagree, Not applicable/I don't know.

I ask for assistance when I need it.

I balance personal and professional demands.

I am able to negotiate in complex situations.

I am internally driven.

I have a plan for achieving my academic career goals.

I have a tolerance for change.

I have input into how I spent my time.

I sought out a mentor.

I personally see more opportunities than challenges.

I set appropriate boundaries to maintain productivity.

I routinely solicit feedback on my professional growth.

I considered leaving academic medicine.

Over the lastacademic year, about how many times have you participated in professional development activities (e.g., workshops, conferences, online tutorials) related to the following areas? Response options: 0, 1-3, 4-6, 7-9, More than 10, Not applicable/I don't know.

Promotion and tenure

Teaching and learning

Research 


\section{Leadership}

Advancement of women

Advancement of underrepresented minorities

Other diversity issues

To what extent are you currently engaged in the following activities? Response options: Very much, Quite a bit, Some, Very little, Not applicable/I don't know.

Professional organization(s) in your field

Mentoring colleagues

Committee work at the school or campus level

Collaborations with colleagues in my primary unit

Serving as a mentor to learners

Faculty development activities

Given the expectations in your primary unit, how do you currently rate yourself? Response options: Well below, Slightly below, At expectations, Slightly above, Well above.

Relative value units (RVUs) benchmarks

Securing external funding

Teaching evaluations

Number of peer-reviewed publications

Number of peer-reviewed conference presentations

Number of learners with whom I interact

Participation in university/department/school service

Participation in professional organizations in my field

Translating research into practice

Consider your experiences over the last academic year, and please rate your level of satisfaction with the following items: Very satisfied, Somewhat satisfied, Neither satisfied nor dissatisfied, Somewhat dissatisfied, Very dissatisfied, Not applicable/I don't know.

Efforts to promote diversity in my primary unit

Sense of community in my primary unit

Promotion and tenure process

My overall level of productivity Overall, how satisfied are you with your career? Response options: Very satisfied, Somewhat satisfied, Neither satisfied nor dissatisfied, Somewhat dissatisfied, Very dissatisfied, Not applicable/l don't know. Responses to the following demographic items will be reported only in the aggregate, and no individual respondent will be identifiable.

School

Division

Department 
Enter the number of years as faculty at your current institution. Write in.

Enter the number of years as faculty at any other institution. Write in.

Degree(s). Response options: DDS, MD, PhD, MBA, MPH, MA/ MS, MLS, RN, BSN, MSN, Other (write in).

Track: Tenure track, Clinical (non-tenure track), Lecturer, Academic Specialist, Research/scientist track, Librarian, Other.

Faculty Rank: Assistant, Associate, Full Professor, Other.

Gender: Response options: Male, Female.

Race/ethnicity. Response options: American Indian or other Native American; Asian, Asian American, or Pacific Islander; black or African American; white (non-Hispanic); Mexican or Mexican American; Puerto Rican; other Hispanic or Latino; multiracial; Other; I prefer not to respond.

Employment status. Response options: Full time, Part time.

If you have any additional comments you would like to make, please type them below.

\section{REFERENCES}

Baldwin, R. G. (1990). Faculty vitality beyond the research university. Journal of Higher Education, 61(2), 160-180.

Barratt, M. S., \& Moyer, V. A. (2004). Effect of a teaching skills program on faculty skills and confidence. Ambulatory Pediatrics, 4 (1 Suppl.), 117-120.

Berbano, E. P., Browning, R., Pangaro, L., \& Jackson, J. L. (2006). The impact of the Stanford Faculty Development Program on ambulatory teaching behavior. Journal of General Internal Medicine, 21(5), 430-434.

Birch, A. J., \& Gray, T. (2009). Ten ways to use a relational database at a faculty development center. In L. B. Nilson \& J. E. Miller (Eds.), To improve the academy: Resources for faculty, instructional, and organizational development, Vol. 27 (pp. 72-87). San Francisco, CA: Jossey Bass.

Bland, C. J., Seaquist, E., Pacala, J. T., Center, B. A., \& Finstad, D. (2002). One school's strategy to assess and improve the vitality of its faculty. Academic Medicine, 77(5), 368-376.

Bland, C. J., Weber-Main, A. M., Lund, S. M., \& Finstad, D. A. (2005). The research-productive department: Strategies from departments that excel. San Francisco, CA: Jossey-Bass/Anker. 
Bothell, T. W., \& Henderson, T. (2004). Evaluating the return on investment of faculty development. In C. M. Wehlburg \& S. Chadwick-Blossey (Eds.), To improve the academy: Resources for faculty, instructional, and organizational development, Vol. 22 (pp. 52-70). Bolton, MA: Anker.

Castillo-Page, L. (2012). Diversity in medical education: Facts and figures 2012. Association of American Medical Colleges. Retrieved from https://www .aamc.org/initiatives/diversity/

Chan, S. S., \& Burton, J. (1995). Faculty vitality in the comprehensive university: Changing contexts and concerns. Research in Higher Education, 36(2), 219-34.

Chism, N.V.N., \& Szabo, B. (1997-98). How faculty development programs evaluate their services. Journal of Staff. Program, and Organizational Development, 15(2), 55-62.

Clark, S. M., Boyer, C. M., \& Corcoran, M. (1985). Faculty and institutional vitality in higher education. In S. M. Clark \& D. Lewis (Eds.), Faculty vitality and institutional productivity: Critical perspectives for higher education (pp. 3-24). New York, NY: Teachers College Press.

Clark, S. M., \& Lewis, D. R. (Eds.). (1985). Faculty vitality and institutional productivity: Critical Perspectives for higher education. New York, NY: Teachers College Press.

Dankoski, M. E., Palmer, M. M., Banks, J., Brutkiewicz, R. R., Walvoord, E., Hoffmann-Longtin, K., Bogdewic, S. P., \& Gopen, G. D. (2012). Academic writing: Supporting faculty in a critical competency for success. Journal of Faculty Development, 26(2), 47-54.

Dankoski, M. E., Palmer, M. M., Nelson-Laird, T., Garver, A., \& Bogdewic, S. P. (2012). An expanded model of faculty vitality in academic medicine. Advances in Health Science Education, 17(5), 633-649.

Joliff, L., Leadley, J., Coakley, E., \& Sloane R. A. (2012). Women in U.S. academic medicine and science: Statistics and benchmarking report 2011-2012. Association of American Medical Colleges. Retrieved from https://www.aamc.org/members/gwims/statistics/

Kelly, A. M., Cronin, P., \& Dunnick, N. R. (2007). Junior faculty satisfaction in a large academic radiology department. Academic Radiology, 14(4), 445-454.

Knight, A. M., Cole, K. A., Kern, D. E., Barker, L. R., Kolodner, K., \& Wright, S. M. (2005). Long-term follow-up of a longitudinal faculty development program in teaching skills. Journal of General Internal Medicine, 20(8), 721-725.

Lief, S., Baker, L., Mori, B., Egan-Lee, E., Chin, K., \& Reeves, S. (2012). Who am I? Key influences on the formation of academic identity within a faculty development program. Medical Teacher, 34(3), e208-215. 
Morzinski, J. A., \& Fisher, J. C. (2002). A nationwide study of the influence of faculty development programs on colleague relationships. Academic Medicine, 77(5), 402-406.

Morzinski, J. A., \& Simpson, D. E. (2003). Outcomes of a comprehensive faculty development program for local, full-time faculty. Family Medicine, 35, 434-439.

Palmer, M. M., Dankoski, M. E., Smith, J. S., Brutkiewicz, R. R., \& Bogdewic, S. P. (2011). Exploring changes in culture and vitality: The outcomes of faculty development. Journal of Faculty Development, 25(1), 21-27.

Pololi, L., Conrad, P., Knight, S., \& Carr, P. (2009). A study of the relational aspects of the culture of academic medicine. Academic Medicine, 84(1), 106-114.

Pololi, L. H., \& Frankel, R. M. (2005). Humanizing medical education through faculty development: Linking self-awareness and teaching skills. Medical Education, 39(2), 154-162.

Pololi, L. H., Knight, S., Dennis, K., \& Frankel, R. (2002). Helping medical school faculty realize their dreams: An innovative, collaborative mentoring program. Academic Medicine, 77(5), 377-384.

Ries, A., Wingard, D., Gamst, A., Larssen, C., Farrell, E., \& Reznik, V. (2012). Measuring faculty retention and success in academic medicine. Academic Medicine, 87(8), 1046-1051.

Schindler, B. A., Novack, D. H., Cohen, D. G., Yager, J., Wang, D., Shaheen, N. J., \& Drossman, D. A. (2006). The impact of the changing health care environment on the health and well-being of faculty at four medical schools. Academic Medicine, 81(1), 27-34.

Simpson, D. E., Bragg, D., Biernat, K., \& Treat, R. (2004). Outcomes results from the evaluation of the APA/HRSA Faculty Scholars Program. Ambulatory Pediatrics, 4 (1 Suppl.), 103-112.

Smith, M. A., Barry, H. C., Dunn, R. A., Keefe, C., \& Weismantel, D. (2006). Breaking through the glass ceiling: A survey of promotion rates of graduates of a primary care faculty development fellowship program. Family Medicine, 38(7), 505-510.

Steinert, Y., Naismith, L., \& Mann, K. (2012). Faculty development initiatives designed to promote leadership in medical education. A BEME systemic review. BEME Guide 19. Medical Teacher, 34(6), 483-502.

Wingard, D. L., Garman, K. A., \& Reznik, V. (2004). Facilitating faculty success: Outcomes and cost benefit of the UCSD National Center of Leadership in Academic Medicine. Academic Medicine, 79 (10 Suppl.), S9-S11.

Woods, S. E., Reid, A., Arndt, J. E., Curtis, P., \& Stritter, F. T. (1997). Collegial networking and faculty vitality. Family Medicine, 29, 45-49. 